ISSN 0103-5150

Fisioter. Mov., Curitiba, v. 25, n. 1, p. 141-151, jan./mar. 2012 Licenciado sob uma Licença Creative Commons

\title{
Análise epistemológica dos estudos de conclusão de curso nos programas de Pós-Graduação com Doutorado do Brasil
}

\author{
Epistemological analysis of thesis and dissertations from \\ Postgraduate programs with Doctoral degree in Brazil
}

\author{
Alessandro Haupenthal $^{[\mathrm{a}]}$, Janeisa Virtuoso ${ }^{[\mathrm{b}]}$, Natalia Duarte ${ }^{[\mathrm{b}]}$, Daniela Pacheco dos Santos ${ }^{[\mathrm{b}]}$, \\ Alexandro Andrade ${ }^{[c]}$ \\ [a] Estudante de Doutorado, Centro de Ciências da Saúde e do Esporte (CEFID), Universidade do Estado de Santa Catarina \\ (UDESC), Florianópolis, SC - Brasil, e-mail: dedsnet@yahoo.com.br \\ [b] Estudante de Mestrado, CEFID, UDESC, Florianópolis, SC - Brasil. \\ [c] Professor Doutor, CEFID, UDESC, Florianópolis, SC - Brasil.
}

\section{Resumo}

Objetivos: Analisar a produção de conhecimento voltada à área de fisioterapia vinculada aos trabalhos de conclusão da Pós-Graduação: Mestrado e Doutorado. Materiais e métodos: Este estudo descritivo usou o método nomeado análise de conteúdo com as seguintes categorias de análise: disciplina e palavras-chave. Foram analisados nos estudos os descritores com ocorrência maior que cinco vezes, de acordo com a frequência observada no programa TextSTAT. A partir desses critérios foram selecionados os seguintes programas: Ciências da Reabilitação da Universidade de Minas Gerais (UFMG), Fisioterapia da Universidade Federal de São Carlos (UFSCar) e Ciências da Reabilitação da Universidade de São Paulo (USP). Foram analisadas as produções disponíveis na internet desses programas de Pós-Graduação. Resultados: Foram registrados 219 trabalhos de conclusão de curso, nos quais Ortopedia foi a disciplina mais prevalente, seguida de Respiratória e de Pediatria. Quanto aos descritores, o exercício físico teve maior destaque, seguido de idoso, fisioterapia e marcha. Conclusão: Ao analisar parte da produção de conhecimento voltada à área de fisioterapia, destaca-se a ocorrência da disciplina "Ortopedia", refletindo maior produção científica, já que essa é a área de maior atuação profissional. Os trabalhos podem ser caracterizados como unidirecionais, englobando geralmente uma área do conhecimento e não dando abertura para diferentes visões sob o mesmo 
aspecto. Além disso, existe forte ausência de estudos visando ao aspecto social, sendo que estes praticamente não existiram nos estudos analisados.

Palavras-chave: Conhecimento. Pesquisa. Dissertações. Teses. Epistemologia.

\section{Abstract}

Objectives: To analyze knowledge production related to physiotherapy through the analysis of Postgraduate thesis and dissertation. Materials e methods: This descriptive study is a content analysis based on two categories: subjects and keywords. Keywords were analyzed only if they appeared more than 5 times, this frequency was verified through TextSTAT software. The following postgraduate courses were included: Science of Rehabilitation from University of Minas Gerais (UFMG), Physiotherapy from Federal University of São Carlos (UFSCar) and Science of Rehabilitation from University of São Paulo (USP). All studies from these Postgraduate programs available on internet sites were analyzed. Results: We verified 219 studies, Orthopedics was the most prevalent subject, followed by Pulmonology and Pediatrics. In relation to keywords, physical activity presented the highest prevalence, followed by elderly, physiotherapy and gait. Conclusion: After analyzing part of the knowledge production in physiotherapy, we concluded that Orthopedics subject is in evidence, this reflects a greater production in this area which is also considered the largest professional area. The studies could be characterized as one dimensional since it generally envolves only one knowledge area, not allowing other point of views to interfere in the same analyzed aspect. Furtheremore, there seems to be a scarcity of studies related to social aspects, since no studies related to this topic were found.

Keywords: Knowledge. Research. Dissertation. Thesis. Epistemology.

\section{Introdução}

No Brasil, em 1951, surgiu o primeiro curso de Fisioterapia, cujo objetivo era formar técnicos em fisioterapia. Suas instalações eram no sétimo andar do Instituto Central do Hospital das Clínicas em São Paulo e as aulas, ministradas pelo corpo docente de médicos do próprio hospital.

Em 1964 foram criados os cursos superiores de Fisioterapia e de Terapia Ocupacional (1). Segundo Botomé e Rebelatto (2), no fim do século XX a fisioterapia passou a fazer parte da chamada "Área da Saúde" e foi evoluindo no decorrer da história, tendo seus recursos e formas de atuação quase que voltados exclusivamente ao atendimento do indivíduo doente.

O corpo de conhecimentos, ou as formas de trabalho que viriam a caracterizar a fisioterapia a partir de então, parece ter seguido a mesma direção das especialidades médicas, no sentido de compartimentalizar áreas de estudo e campos de atuação profissional $(3,4)$. De acordo com Japiassu (5), cada disciplina, por meio de seu enfoque específico, desenvolve instrumentos para conhecer a realidade e os problemas a partir de um determinado ponto de vista, ou seja, é capaz de revelar uma dimensão do humano. Mas essa visão unidisciplinar invariavelmente fragmenta o objeto e o reduz por privilegiar a decomposição do todo em partes. A ciência em si é reducionista e analítica, mas essa divisão em esferas e ramos no estudo do homem para seu entendimento acaba não sendo desfeita na hora de atuação do profissional (6).

Essa compartimentalização faz-se presente também no conceito de saúde, que nas últimas décadas vem passando por intensas transformações, principalmente no que diz respeito ao modelo de saúde adotado, passando de um modelo biomédico e hospitalocêntrico, curativo e reabilitador, para um modelo holístico e sistêmico. Nesse modelo, as esferas entre promoção, prevenção e recuperação da saúde fazem parte de um processo contínuo, no qual a participação popular e a interdisciplinaridade dos diferentes profissionais faz-se presente como fator importante para o escopo geral e o resultado final desse processo. Nesse sentido, entende-se saúde não como o avesso da doença, mas como a busca do equilíbrio do ser humano, devendo, portanto, romper os estreitos limites da assistência curativa (7-9).

Faz alguns anos que a visão da saúde passou da mera ausência da doença para a noção de bem-estar físico e mental, e daí para conceito mais amplo, que 
inclui adequação de vida social (8-10). Ocorre claramente uma mudança de paradigma, inclusive com ruptura semântica entre o conceito atual de saúde e o anterior. Modifica-se a prática sanitária, passando-se da antiga (curativista) para a atual - a vigilância da saúde. Essa visão sobre paradigma surge com Fleck $(11,12)$ e, posteriormente, reforçada e popularizada por Kuhn (13), pode ser entendida como o conjunto de elementos culturais, conhecimentos e códigos teóricos, técnicos ou metodológicos, compartilhados pelos membros de uma comunidade científica $(14,15)$. Esses autores focam a visão social para a produção e certificação do conhecimento, mas apesar do aspecto social ser levado em consideração para a criação e aceitação do conhecimento, o mesmo aspecto social muitas vezes é deixado de lado na atuação profissional que, dessa forma, passa a ser focada individualmente no paciente (16).

A atuação do fisioterapeuta hoje deve prever a resolutividade das necessidades de saúde que extrapolam a esfera de intervenção curativa e reabilitadora individual, por meio da promoção da saúde, prevenção de doenças e educação continuada (17, 18). De acordo com Demo (19), a possibilidade de uma compreensão integral do ser humano e do processo saúde-doença, objeto do trabalho em saúde, passa necessariamente por uma abordagem interdisciplinar (20). Com o desenvolvimento das sociedades tendo em perspectiva a interconexão do conhecimento, buscando cada vez mais a instituição de dimensões mais ampliativas e globalizantes nas esferas do saber e do fazer, a tendência da disciplinaridade é ser transcendida $(6,19,21)$.

A interdisciplinaridade caracteriza-se pela intensidade das trocas entre os especialistas e pelo grau de integração real das disciplinas no interior de um mesmo projeto de pesquisa $(6,20,22)$. Dentro desse contexto de alteração de paradigma ligado à saúde e visão interdisciplinar para o profissional, este estudo objetivou verificar, por meio da produção da Pós-Graduação em Fisioterapia, quais os temas de interesse que esses profissionais estão focando em seus estudos.

\section{Materiais e métodos}

Este estudo pode ser caracterizado como descritivo, com uma abordagem por meio da análise de conteúdo (23). Para analisar a produção de conhecimento em fisioterapia, optou-se por selecionar os programas de Pós-Graduação relacionados à fisioterapia que possuem Mestrado e Doutorado. A partir desses critérios, foram selecionados os seguintes programas: Ciências da Reabilitação da Universidade de Minas Gerais (UFMG), Fisioterapia da Universidade Federal de São Carlos (UFSCar) e Ciências da Reabilitação da Universidade de São Paulo (USP). Foram analisadas as produções disponíveis na internet desses programas de Pós-Graduação.

Em um primeiro instante foi realizada a fase de leitura flutuante, que serviu para efetivar o levantamento das categorias de análise relacionadas com as disciplinas pertencentes à fisioterapia: Ortopedia, Respiratória, Cardiologia, Neurologia, Reumatologia, Pediatria, Desportiva, Ginecologia, Preventiva, Saúde Pública, Dermato-Funcional e Eletroterapia.

A partir do corpus do estudo, foram criadas fichas de preenchimento que continham o título do estudo, as palavras-chave, os autores e a categoria de análise. Essa planilha foi salva no formato ".txt". Dessa forma, a análise de dados pode ser efetuada pelo programa TextSTAT.

A partir dos estudos selecionados foi utilizado o programa TextSTAT para a contagem das palavras-chave e para a caracterização de sua coocorrência. As palavras-chave foram analisadas a partir da frequência simples com uma ocorrência maior que cinco vezes. Para a coocorrência foram analisadas as principais ligações de cada uma das palavras com as disciplinas correspondentes para efetuação de um organograma. Tanto para a frequência como para a coocorrência o programa considera qualquer diferença na palavra como diferença real, exemplo: análise é diferente de Análise, dessa forma essas palavras, que são iguais em seu conteúdo, mas diferentes somente na grafia, foram somadas e consideradas a mesma.

\section{Resultados}

Foram analisadas 219 dissertações e teses. 0 resultado da frequência em relação às palavras-chave pode ser visto no Gráfico 1. Destaca-se o uso dos termos "exercício físico", "idoso", "fisioterapia" e "marcha" como os mais frequentes nos trabalhos de Pós- Graduação analisados.

Essas palavras-chave foram organizadas em um organograma, em relação à sua ocorrência, por 


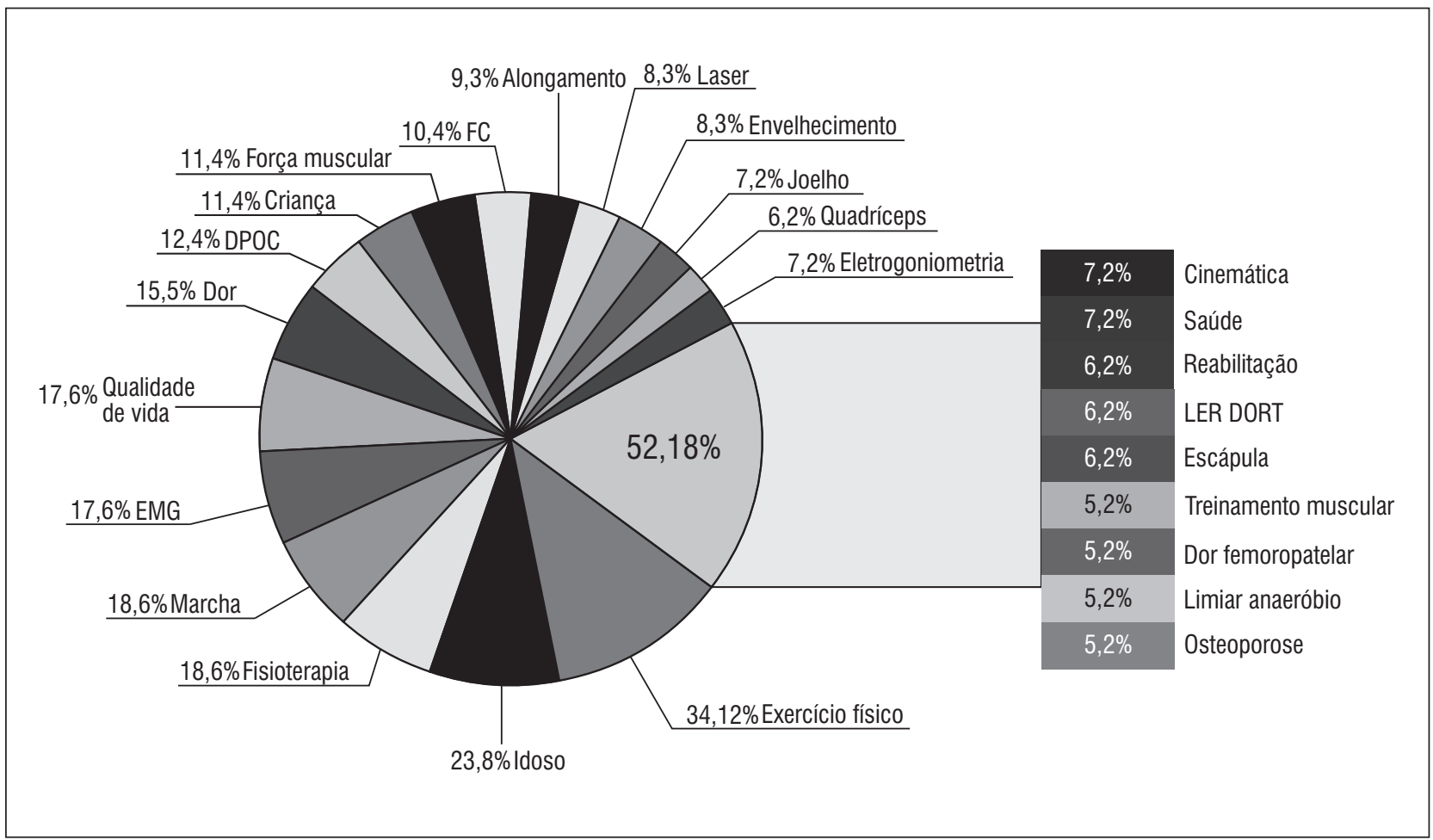

Gráfico 1 - Gráfico representativo da frequência (\%) e ocorrência (número de vezes) das palavras-chave nos estudos analisados Nota: Destaca-se um cuidado na leitura deste gráfico: a área cinza clara, 52; 18\%, representa o somatório dos percentuais que seriam muito pequenos para serem representados individualmente no gráfico pizza.

Fonte: Dados da pesquisa.

programa de Pós-Graduação (Figura 1). Novamente, em cada um dos programas as mesmas palavras-chave continuaram aparecendo com maior frequência.

Quanto às categorias de análise, a mais frequente foi a Ortopedia, duas vezes maior que a segunda colocada (Respiratória) e a terceira (Pediatria) (Gráfico 2).

Para relacionar as categorias de análise com as palavras-chave e analisar dentro de cada categoria qual o foco dos estudos, foi criada a Figura 2. Nessa figura o tamanho da palavra representa a importância do termo para a fisioterapia; abaixo de cada categoria estão as palavras-chave que predominam dentro dessa categoria. Pode ser visto que a Ortopedia é relacionada principalmente com exercício físico, eletromiografia e treinamento muscular. Nos estudos de Respiratória, além do exercício físico destacam-se o idoso e a doença pulmonar obstrutiva crônica. Para Pediatria aparece desenvolvimento, criança e linguagem. Assim, quando analisadas separadamente, as palavras-chave por categoria são diferentes daquela no geral. Nomeamos essa figura de "pirâmide do conhecimento em fisioterapia”, já que apresenta um retrato da produção e seu foco para os programas de Pós-Graduação no Brasil.

\section{Discussão}

O corpo de conhecimento de uma profissão é a chave para o delineamento e a descrição dessa profissão $(10,24,25)$. A produção desse conhecimento é responsabilidade de cada grupo de profissionais (10). Ess corpo de conhecimento gerado é essencial para raciocinar e tomar decisões o que é o centro da prática profissional $(10,24)$. Ainda em relação a esse corpo de conhecimento, dois fatores fundamentais devem ser destacados: a) sua relação com o processo social de formação e b) construção e não descoberta. 0 primeiro desses fatores é relacionado ao processo de construção dessa base, nomeada corpo de conhecimento. Esse processo caracteriza-se essencialmente como processo social de construção dos paradigmas aceitos pelo grupo profissional em questão $(10,13)$. 0 segundo é relacionado a como cada indivíduo cria sua construção e interpretação 
a partir de suas bases individuais de conhecimento e experiência prática; enfim, de que forma ele se apropria e utiliza o conhecimento existente (10).
A epistemologia é o ramo da filosofia que estuda os diferentes tipos de conhecimento, ainda mais qual o critério que pode e é utilizado para

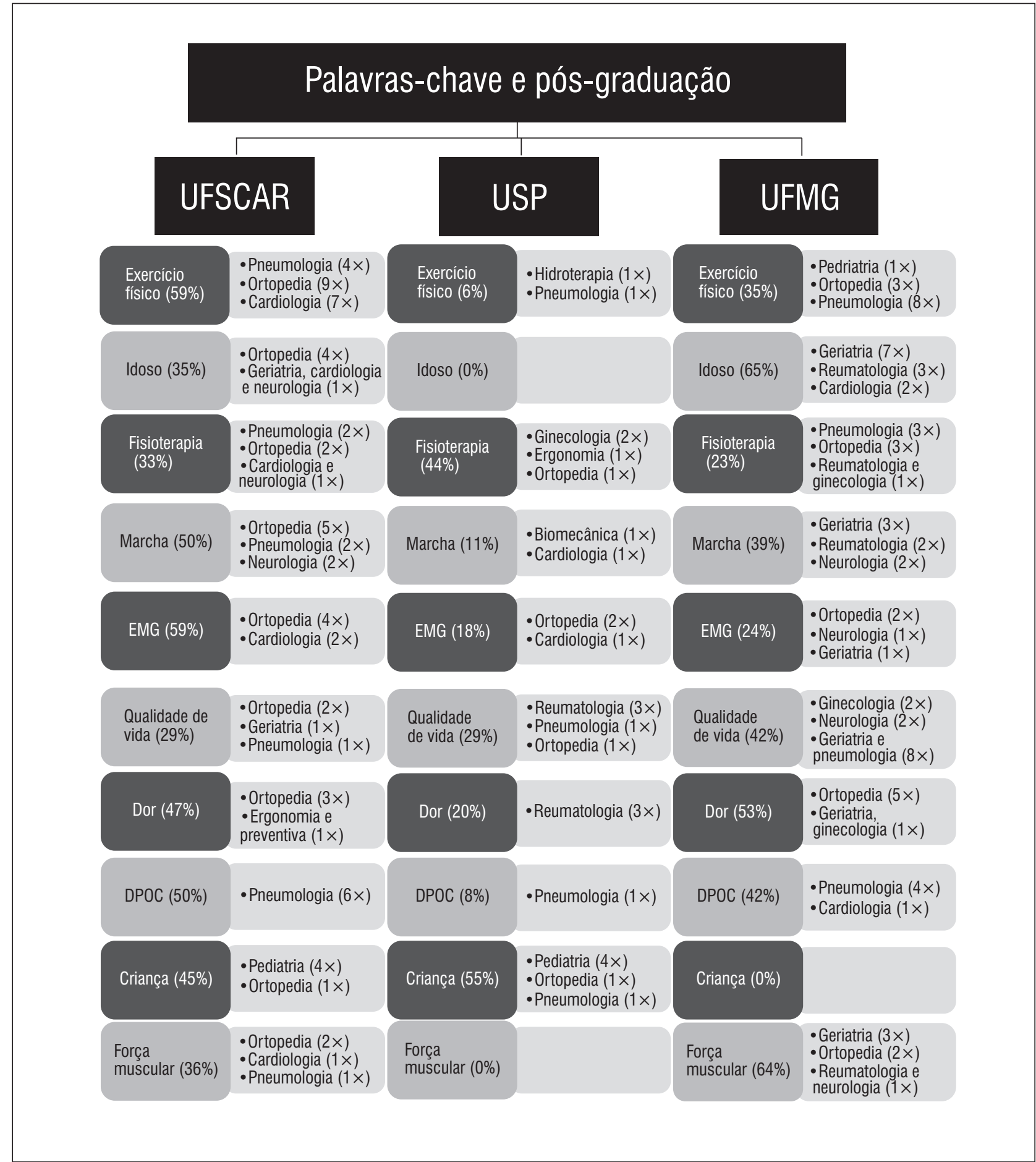

Figura 1 - Organograma representativo da relação entre as palavras-chave e o programa de Pós-Graduação.

Nota: Foram listadas as dez palavras-chave mais frequentes. 0 percentual (\%) representa a frequência da palavra em determinado programa de Pós-Graduação em relação ao total produzido pelos programas. Acompanhando a palavra-chave estão as três disciplinas mais frequentemente relacionadas e o número de vezes $(X)$ que essa relação ocorreu.

Fonte: Dados da pesquisa. 


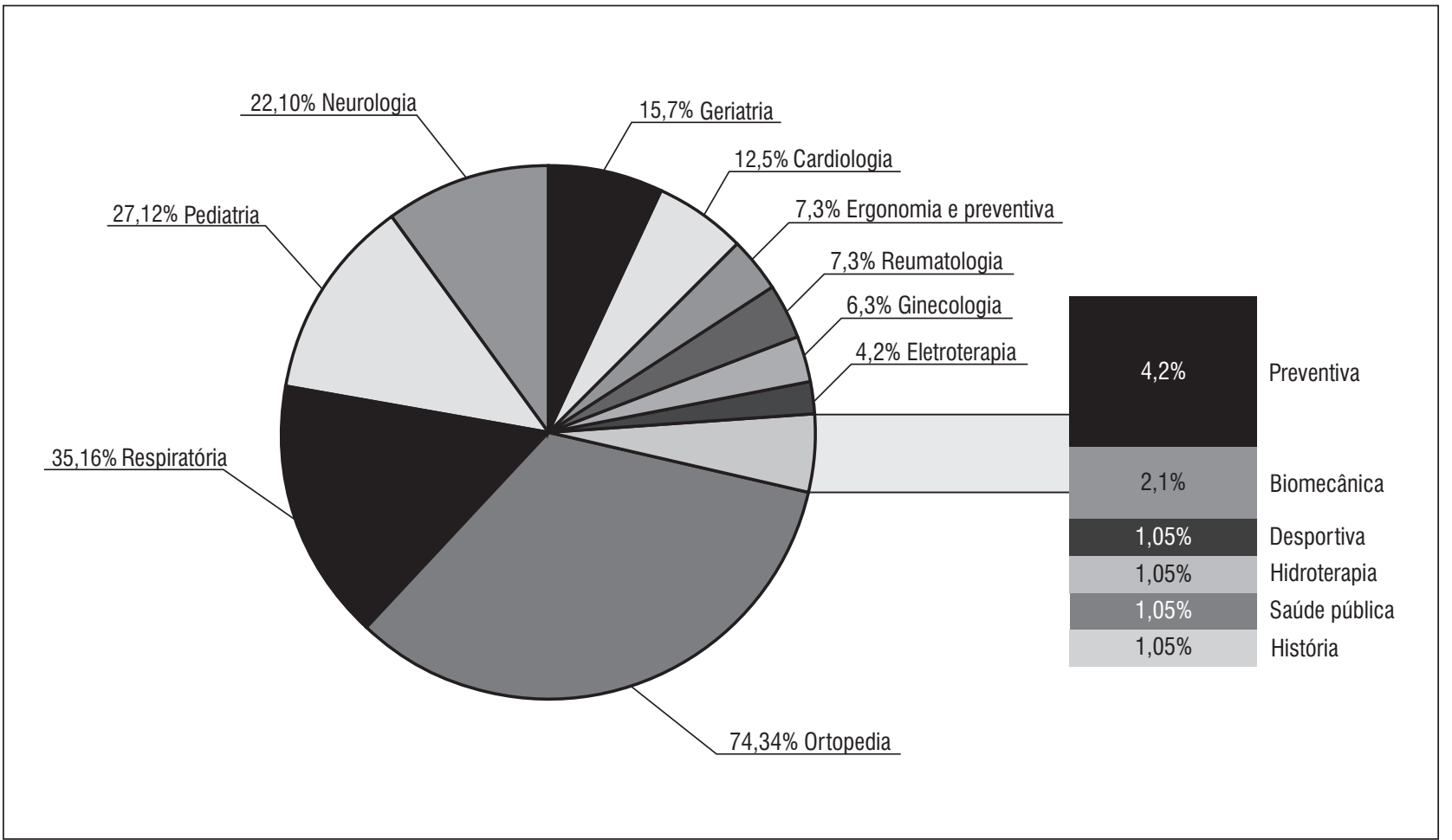

Gráfico 2 - Gráfico representativo da frequência (\%) e ocorrência (número de vezes) da categoria de análise nomeada disciplina nos estudos analisados

Nota: Destaca-se um cuidado na leitura deste gráfico: a área cinza clara, 10; 5\%, representa o somatório dos percentuais que seriam muito pequenos para serem representados individualmente no gráfico pizza.

Fonte: Dados da pesquisa.

diferenciar entre o conhecimento e o não conhecimento (10). Com isso, o termo conhecimento implica uma noção que é julgada para atingir algum padrão, sendo dependente do paradigma (visão ou epistemologia) adotado pela pessoa que julga essa noção. A partir dessa caracterização criou-se uma divergência de pensamento: a) em Higgs e Titchen (10) pode ser visto que é importante o conhecimento das diferentes visões (paradigmas) para a escolha de qual representaria melhor a realidade do fisioterapeuta e em b) Robertson (26) destaca que a fisioterapia é uma profissão clínica e como tal deve focar seus esforços nos métodos de desenvolver e explicar o conhecimento na clínica e no domínio clinicamente relevante.

Em Kuhn (13) tem-se o relato de que as ciências que possuem sua razão de ser em uma necessidade social exterior não têm a necessidade de assumir um paradigma único para seu desenvolvimento. Robertson (26) está correta em relatar que conhecer mais sobre a epistemologia não vai aumentar a base de conhecimento na área, mais que isso, a epistemologia não serve para a produção instrumental, mas sim para pensar sobre o devir (para onde vamos) e qual a base de conhecimento que aceitamos para levarmos nesse processo. Sendo a fisioterapia uma área aplicada, acredita-se que não deva assumir um paradigma único, mas, para poder se situar entre tudo o que existe atualmente, a epistemologia e a filosofia da ciência podem ser importantes ferramentas para o fisioterapeuta entender o contexto ao qual está presente.

Um problema que ainda está presente é que, por se caracterizar como área prática, a fisioterapia tinha pouco de seus métodos bem estruturados e embasados $(10,26)$. Contudo, essa falta de relatos não quer dizer que o tratamento não seja efetivo, mas simplesmente que existe pouca evidência de que ele seja. Em Robertson (26) pode ser visto que nessa época era estimado que menos de $1 \%$ dos fisioterapeutas estavam escrevendo e eram publicados. Além desse processo, como a fisioterapia tem sua história ligada à medicina, as áreas de especialidades também se fazem correlatas. 


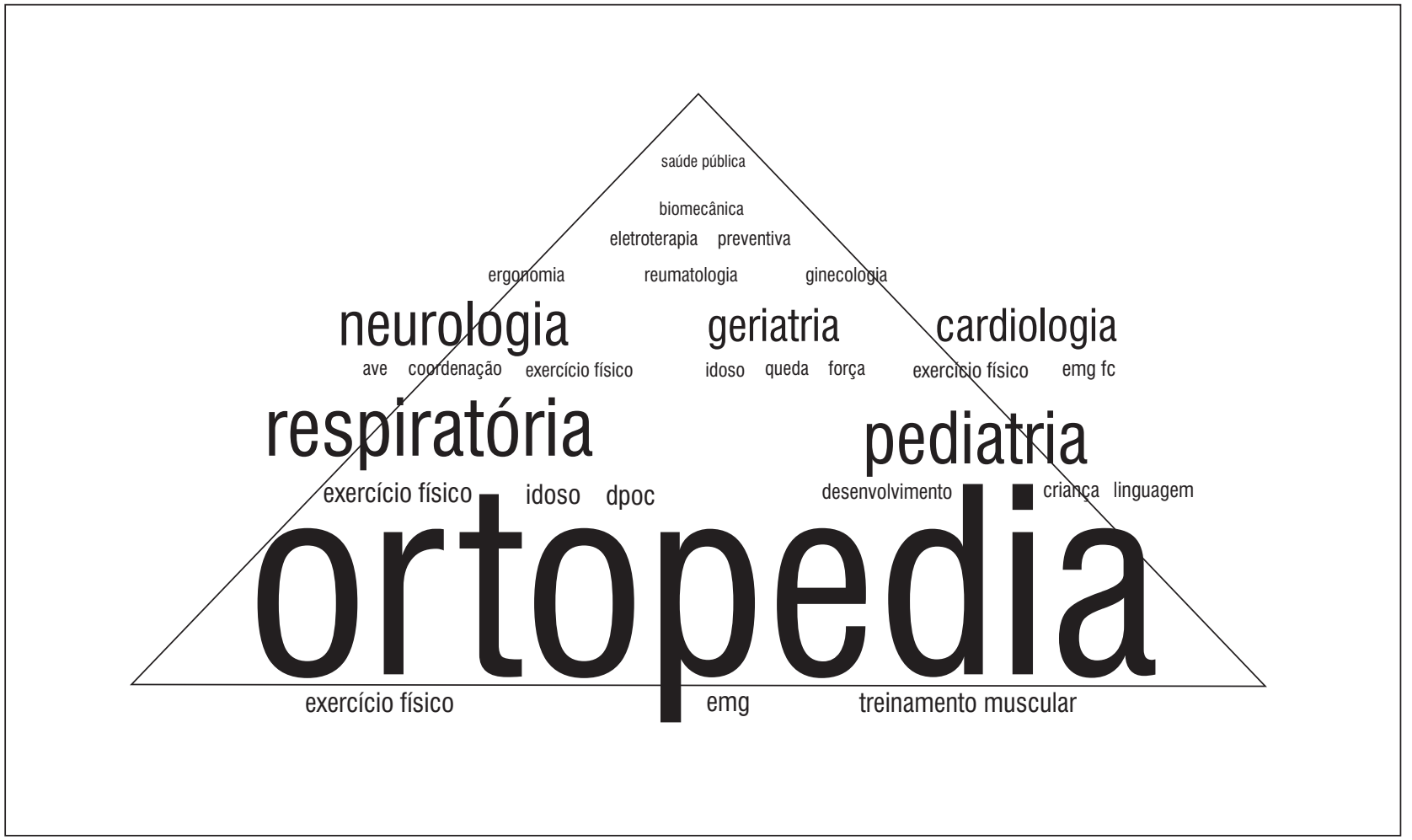

Figura 2 - Pirâmide da base do conhecimento produzido em fisioterapia a partir dos trabalhos de conclusão de curso de Pós-Graduação. 0 tamanho das palavras representa sua importância em termos de produção em relação ao total das diferentes disciplinas Nota: Abaixo de cada disciplina, estão relacionadas as três mais frequentes palavras relacionadas à disciplina escrita acima.

Fonte: Dados da pesquisa.

A partir dessa contextualização pode ser entendido o porquê dos resultados apresentados neste estudo, nos quais as áreas de estudo da fisioterapia são decorrentes de subespecialidades e com o foco para a efetividade do tratamento. Efetividade essa que é amplamente abordada com a chamada fisioterapia ou prática baseada em evidência (27-30), que preconiza a busca pela maior evidência científica para embasar as ações profissionais. Mas está se esquecendo de que, ao mesmo tempo, ela preconiza a reavaliação crítica de nossas ações e, além disso, defende que devem ser respeitadas as escolhas do paciente e entendido seu processo mental e cultural para que possa haver uma negociação para as ações serem realizadas em conjunto $(25,30)$.

Alguns pesquisadores analisaram a prática baseada em evidência em relação a seu uso pelos profissionais $(31,32)$. Com essa abordagem, além da efetividade do tratamento, começou a ser discutida também a lacuna entre o conhecimento e as pesquisas na universidade e as competências requeridas para a prática profissional $(10,25,33)$. Em Jette et al. (31) e em Connolly et al. (34) vê-se que a aceitação da pesquisa na prática do dia a dia profissional ainda é baixa. Em 1987, Lehmkuhl (35) já relatava a preocupação com o delineamento do estudo desde sua pergunta inicial até sua conclusão para sua posterior aplicação na prática diária. Esta ainda é uma preocupação atual: fazer com que o que se produz na pesquisa seja importante para aos profissionais, e fazer com que os profissionais saibam como buscar essa informação e utilizá-la na sua rotina (10, $30,36,37)$. Dentro desse contexto os autores relatam também que deve existir a facilidade de acesso e compreensão do que é escrito, facilidade de acesso essa que, atualmente, para os estudantes e alguns profissionais torna-se difícil. Uma vez que, pela competição entre visibilidade e fator de impacto, muitos pesquisadores optam por publicar artigos somente ou principalmente no exterior, limitando o corpo de profissionais que não dominam outra língua ou não têm acesso a essas bases de dados. Assim, cabe uma 
política pública para aumentar o número de revistas nacionais com boa indexação e índices para que os pesquisadores queiram publicar em sua própria língua, facilitando o acesso aos estudos.

Para Morin (6), a disciplina é uma categoria organizadora dentro do conhecimento científico; ela institui a divisão e a especialização do trabalho e responde à diversidade das áreas que as ciências abrangem. Uma disciplina tende naturalmente à autonomia pela delimitação das fronteiras, da linguagem em que ela se constitui, das técnicas que é levada a elaborar e a utilizar e, eventualmente, pelas teorias que lhe são próprias.

Quase metade das palavras-chave mais frequentes $(49,8 \%)$ se encontra em apenas duas disciplinas, a Ortopedia e a Respiratória. Esse resultado mostra que, além das duas áreas serem grandes campos de pesquisa em fisioterapia, a interdisciplinaridade ainda é escassa nos projetos de pesquisa. Uma disciplina não é suficiente para suprir todos os problemas tocantes a ela, pois há noções que circulam e, com frequência, atravessam clandestinamente as fronteiras, sem serem detectadas. Afinal, noção alguma é restrita a um único campo disciplinar, mas todas são "noções migradoras" $(6,20)$.

A grande frequência de aparecimento da palavra exercício físico já poderia ser esperada, em razão da grande relação da área com a ciência do movimento (38), pois um dos maiores instrumentos de trabalho de um fisioterapeuta é a cinesioterapia. Doze dos 34 aparecimentos dessa palavra-chave corresponderam à disciplina de Ortopedia.

Uma base de dados mundial de evidências em fisioterapia (PEDro), que contém mais de 15.000 ensaios clínicos randomizados, revisões sistemáticas e diretrizes de prática clínica em fisioterapia, avalia de forma independente a qualidade dos estudos, por uma pontuação que varia de 0 a 10 , demonstrando que quanto maior a nota maior a evidência científica de bons resultados de um procedimento clínico. A palavra-chave "exercício físico" foi consultada, e as maiores evidências encontradas (nota 9) foram do exercício físico para dor lombar, capsulite adesiva e dor fêmoro-patelar. Assim, parece ser uma tendência mundial o grande número de estudos na área de Ortopedia, o que vem consolidando a prática clínica nessa disciplina.

Contudo, fica claro que as pesquisas mais frequentes e de maior evidência científica ainda estão no âmbito da doença, principalmente porque, dentre 219 trabalhos avaliados, são raros os que abordam as disciplinas relacionadas com prevenção ou promoção de saúde ou sequer com a saúde pública. Além disso, a palavra "saúde" aparece apenas em 2,5\% dos trabalhos.

No campo do ensino, devemos não apenas formar profissionais aptos ao diálogo técnico e leigo com os mais variados setores $(14,39,40)$, como também os professores e os alunos devem estar habilitados a desempenhar atividades antes não requeridas, como a atuação política junto a grupos populacionais, institucionais e órgãos de administração pública, de forma a poder atender às solicitações que a sociedade encaminha para respostas técnicas atualizadas para os velhos e os novos problemas em saúde e ambiente.

A política de divulgação do conhecimento científico parece, também, não estar sendo valorizada. Uma das palavras-chave mais frequente neste estudo foi "fisioterapia", cujo descritor é amplo e não reflete o foco da pesquisa. Os autores, ao escolherem palavras-chave precisam estar cientes de que estas precisam divulgar com precisão seus resultados e do que se trata o estudo.

A partir dos resultados encontrados pode ser caracterizado que existem áreas básicas para a profissão que carecem de maior atenção. Como exemplos, podem ser citadas a pedagogia, a sociologia e a filosofia. Entende-se esse processo da falta de produção nessas áreas em razão da não importância dada durante o processo de ensino. Uma visão atual do processo de ensino e de um projeto político pedagógico levando em conta aspectos da complexidade pode ser visto em Signorelli et al. (41). A partir dessa perspectiva e conforme destaca Freire (42), não podemos cobrar dos alunos o que não lhes é ensinado, não poderiam ser esperados trabalhos com áreas que não são focadas durante o ensino. Ainda mais, pode ser caracterizado que existe uma falta de preocupação com o ensino a partir dos professores, que ora se veem como fisioterapeutas e ora como pesquisadores, raramente como professores (43). Esse é um fato que deve ser alterado, são os professores que irão formar e moldar por meio da educação os próximos fisioterapeutas, que serão os responsáveis pelo futuro da profissão.

Entender e teorizar a história de seu surgimento e da produção do conhecimento na área facilita a compreensão da identidade profissional e as perspectivas futuras $(44,45)$. Dessa forma, verificando o 
foco dos estudos em fisioterapia e a falta de um papel do vínculo social na produção de conhecimento dessa área $(3,46)$, entende-se que a socialização necessária para o reconhecimento público ainda não foi alcançada $(43,44,47)$. A abordagem social que fica clara nos textos produzidos pelos pesquisadores que pensam o futuro da Pós-Graduação no Brasil $(40,48-51)$ ainda não foi iniciada nos trabalhos de conclusão de curso, refletindo que ainda não está aplicada à pesquisa realizada. Talvez a aplicação dessa abordagem possa ser uma alteração de paradigma para a fisioterapia, como a que ocorreu faz algum tempo quando foi substituído o foco dos estudos da incapacidade para a função, como relata o estudo de Jette (52).

\section{Conclusão}

A partir da análise dos estudos pode ser caracterizado que os trabalhos de conclusão de curso de Pós-Graduação focam seus estudos principalmente na Ortopedia, em seguida na Respiratória e na Pediatria. Quanto à palavra-chave mais frequente, pode ser caracterizado o exercício físico e idoso.

Se combinarmos as duas análises, veremos que a Ortopedia foca seus estudos no exercício físico, eletromiografia e treinamento. A Respiratória observa principalmente exercício físico, idoso e doença pulmonar obstrutiva crônica; e a Pediatria observa desenvolvimento, criança e linguagem.

A partir dessa abordagem fica claro o maior estudo na maior área de atuação profissional, que é a Ortopedia. Os trabalhos ainda podem ser caracterizados como unidirecionais, englobando geralmente uma área do conhecimento e não dando abertura para diferentes visões sob o mesmo aspecto. Além disso, existe forte ausência de estudos visando ao aspecto social, sendo que estes praticamente não existiram nos estudos analisados.

\section{Referências}

1. Sanches E. Preliminar do adestramento de fisioterapeutas no Brasil. Ver Paul Hosp. 1970;4:30-7.

2. Botomé S, Rebelatto J. Fisioterapia no Brasil: fundamentos para uma ação preventiva e perspectivas profissionais. 2nd ed. São Paulo: Manole; 1999.
3. Salmória JG. Uma Aproximação dos Signos - Fisioterapia e Saúde - aos Aspectos Humanos e Sociais. Saúde e Soc. 2008;17(1):73-84.

4. Meyer P, Costa I, Gico V. Ciências sociais e fisioterapia: uma aproximação possível. Hist Ciênc Saúde. 2006;13(4):877-90.

5. Japiassu H. Interdisciplinaridade e patologia do saber. Rio de Janeiro: Imago; 1976.

6. Morin E, Pena-Vega A, Paillard B. Diálogo sobre o conhecimento. São Paulo: Cortez; 2004.

7. Chammé S. Saúde e organização social. Marília: UNESP; 1988.

8. Scliar M. História do Conceito de Saúde. PHYSIS: Rev Saúde Coletiva. 2007;17(1):29-41.

9. Martins A. Novos paradigmas e saúde. PHYSIS: Rev Saúde Coletiva. 1999;9(1):83-112.

10. Higgs J, Titchen A. The nature, generation and verification of knowledge. Physiotherapy. 1995;81(9): 521-30.

11. Löwy I. Ludwig Fleck e a presente história das ciências. Hist Ciênc Saúde. 1994 1(1):17-8.

12. Pfuetzenreiter MR. A epistemologia de Ludwik Fleck como referencial para a pesquisa no ensino na área de saúde. Ciênc \& Educ. 2002;8(2):147-59.

13. Kuhn T. A estrutura das revoluções científicas. 9th ed. São Paulo: Perspectiva; 2009.

14. Santos J, Westphal M. Práticas emergentes de um novo paradigma de saúde: o papel da universidade. Estud Avançad. 1999;13(35):71-88.

15. Higgs J, Titchen A. Research and knowledge. Physiotherapy. 1998;84(2):72-80.

16. Edwards I, Richardson B. Clinical reasoning and population health: Decision making for an emerging paradigm of health care. Physiotherapy. 2008; 24(3):183-93.

17. Pain J, Alves N Filho. Saúde Coletiva: uma nova saúde pública ou campo aberto a novos paradigmas? Rev Saúde Pública. 1998;32(4):299-316.

18. rench HP, Dowds J. An overview of continuing professional development in physiotherapy. Physiotherapy. 2008;94(3):190-7. 
19. Demo P. Desafios modernos da educação. 7 th ed. Rio de Janeiro: Vozes; 1993.

20. Erdmann A, Schlindwien B, Sousa F. REBEn. Rev Bras Enferm. 2006;59(4):560-4.

21. Ferreira RF, Gonzales C. Caminhos da pesquisa e a contemporaneidade. Psicologia: Reflexão e Crítica. 2002;15(2):243-50.

22. Minayo M, Assis S, Deslandes S, Souza E. Possibilidades e dificuldades nas relações entre ciências sociais e epidemiologia. Ciênc Saúde Coletiva. 2003;8(1):97-107.

23. Bardin L. Análise de conteúdo. Lisboa: Edições 70; 1977.

24. Clouder L. Reflective practice: realising its potential. Physiotherapy. 2000;86(10):517-22.

25. Higgs J, Richardson B, Dahlgren M. Developing practice knowledge for health professionals. London: Butterworth-Heinemann; 2004.

26. Robertson VJ. Epistemology, private knowledge, and the real problems in physiotherapy. Physiotherapy. 1996;82(9):534-9.

27. Newham D. Physiotherapy for best effect. Physiotherapy. 1997;83(1):5-11.

28. Dias R, Dias J. Prática baseada em evidências: uma metodologia para a boa prática fisioterapêutica. Fisioter Mov. 2006;19(1):11-6.

29. Marques A, Peccin M. Pesquisa em fisioterapia: a prática baseada em evidências e modelos de estudos. FIsioter \& Pesq. 2005;2(1):43-8.

30. Herbert R, Jamtvedt G, Mead J, Hagen K. Practical evidence-based physiotherapy. London: Elsevier; 2005.

31. Maher CG, Sherrington C, Elkins M, Herbert RD, Moseley AM. Challenges for evidence-based physical therapy: accessing and. PhysTher. 2004;84 (7):644-54.

32. Jette DU, Bacon K, Batty C, Carlson M, Ferland A, Hemingway RD, et al. Evidence-based practice: beliefs, attitudes, knowledge, and behaviors of physical therapists. Phys Ther. 2003;83(9):786-805.

33. Bithell C. Professional knowledge in professional development. Physiotherapy. 1999;85(9):458-9.
34. Connolly B, Lupinnaci N, Bush A. About research in physical therapy. Phys Ther. 2001;81(5):1127-34.

35. Lehmkuhl LDON. Mixing one part common sense with each part statistics in planning the design and reporting the results of clinical research in physical therapy. Phys Ther. 1987;67(12):1851-3.

36. Krebs D, Harris S. Elements of theory presentations in physical therapy. Phys Ther. 1987;68(5):690-3.

37. Tammivaara J, Shepard K. Theory: the guide to clinical practice and research. Phys Ther. 1990;70(9):578-82.

38. Winstein CJ, Knecht HG. Movement science and its relevance to physical therapy. Phys Ther. 1990; 70(12):759-92.

39. Werneck VR. Sobre o processo de construção do conhecimento: o papel do ensino e da pesquisa. Ensaio: Aval Pol Públ Educ. 2006;14(51):173-96.

40. Trindade J, Prigenzi L. Instituições universitárias e produção do conhecimento. São Paulo em Perspec. 2002;16(4):9-14.

41. Signorelli MC, Israel VL, Correa C, Motter A, Takeda $\mathrm{S}$, Gomes A. Um projeto político-pedagógico de graduação em Fisioterapia pautado em três eixos curriculares. Fisioter Mov. 2010;23(2):331-40.

42. Freire P. Pedagogia do oprimido. 49th ed. Rio de Janeiro: Paz e Terra; 2005.

43. Sparkes V. Profession and professionalisation Part 1: role and identity of undergraduate physiotherapy educators and Part 2: Professionalism within academia. Physiotherapy. 2002;88(8):481-492.

44. Nascimento M, Sampaio R, Salmela J, Mancini M, Figueiredo I. A profissionalização da fisioterapia em minas gerais. Rev Bras Fisioter. 2006;10(2):241-7.

45. Rezende M, Moreira M, Amâncio A Filho, Tavares M. A equipe multiprofissional da "Saúde da Família": uma reflexão sobre o papel do fisioterapeuta "Family health" multiprofessional teams: a reflection on the physiotherapist's role. Ciênc e Saúde Coletiva. 2009;14(Supl. 1):1403-10.

46. Bispo J Jr. Formação em fisioterapia no Brasil: reflexões sobre a expansão do ensino e os modelos de formação. Hist Ciênc Saúde. 2009;16(3):655-68.

47. Coury H, Vilella I. Perfil do pesquisador fisioterapeuta brasileiro. Rev Bras Fisioter. 2009;13(4):356-63. 
48. Dantas F. Responsabilidade social e pós-graduação no Brasil: idéias para (avali)ação. Rev Bras Pós Graduação. 2004;1(2):160-72.

49. Neto S, Bevilaque L, Fischer T, Marcovitch J, Almeida V, Nunes E, et al. Mestrado no Brasil: a situação e uma nova perspectiva. Rev Bras Pós Graduação. 2005;2(4):139-44.

50. Guimarães R. O futuro da pós-graduação: avaliando a avaliação. Rev Bras Pós Graduação. 2010; $4(8): 282-92$.
51. Marsiglia R, Spinelli S, Lopes M, Silva T. Das ciências sociais para as ciências sociais em saúde: a produção científica de pós-graduação em ciências sociais. Ciênc Saúde Coletiva. 2003;8(1):275-85.

52. Jette A. Outcomes research: shifting the dominant research paradigm in physical therapy. Phys Ther. 1995;75(11):965-70.

Recebido: 25/01/2011

Received: 01/25/2011

Aprovado: 19/06/2011

Approved: 06/19/2011 